\title{
Current-Induced Spin Accumulation and Spin Transfer Torque in a Néel Domain Wall
}

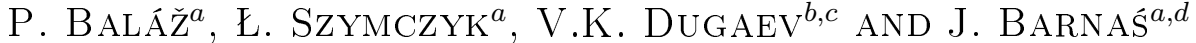 \\ ${ }^{a}$ Department of Physics, Adam Mickiewicz University, Umultowska 85, 61-614 Poznań, Poland \\ ${ }^{b}$ Department of Physics, Rzeszów University of Technology, al. Powstańców Warszawy 6, 35-959 Rzeszów, Poland \\ ${ }^{c}$ Department of Physics \& CFIF, Instituto Superior Técnico, TU Lisbon, av. Rovisco Pais, 1049-001 Lisbon, Portugal \\ ${ }^{d}$ Institute of Molecular Physics, Polish Academy of Sciences, M. Smoluchowskiego 17, 60-179 Poznań, Poland \\ Current-induced spin accumulation in a domain wall and the associated spin torque exerted on the wall \\ are analyzed theoretically. The considerations are limited to a relatively thick Néel domain wall in a bulk \\ ferromagnetic metal. The current-induced spin density is calculated using the linear response theory and the \\ Green function formalism.
}

PACS: $75.60 . \mathrm{Ch}, 75.70 . \mathrm{Cn}$

\section{Introduction}

Domain walls in ferromagnetic metals act like magnetic defects and lead to scattering of conduction electrons. Accordingly, spin density near a domain wall may be different from that far away from the wall, where spin density is spatially uniform. The electron scattering (reflection) from the wall also modifies local conductance of the system, and leads to an additional contribution to the resistance. This contribution is generally positive (domain wall increases the resistance), but surprisingly in some situations (especially for rather thick domain walls) contribution to the resistance from a domain wall may be negative. This is generated by spin accumulation in the domain wall [1]. Thus, spin accumulation created in equilibrium situation (zero current) is important for resistance of the system. This spin accumulation is oriented along the local magnetization associated with a domain wall [1].

Spin accumulation can be significantly modified when current is flowing through the system. This nonequilibrium spin polarization (induced by electric current) is responsible for spin torque exerted on the domain wall. Such a torque vanishes in equilibrium and appears only when current is flowing through the system. It is a consequence of spin transfer from the system of conduction electrons to the system of localized moments responsible for the magnetic domain wall profile. The spin torque appears also in other systems with nonuniform magnetization, like for instance in spin-valve nanopillars, where it can lead to magnetic switching between parallel and antiparallel magnetic configurations $[2,3]$. Such a magnetic reversal has been observed experimentally in many systems, and appears when current density exceeds some threshold value [4].

The current-induced spin torque in systems with domain walls may generally lead to displacements of the walls. Indeed, many recent experiments have demonstrated that the motion of domain walls can be effectively controlled not only by an external magnetic field but also by electric current [5-9]. The domain wall shape becomes modified (in order to reduce the source of the domain wall motion) when current flows through the system and the domain wall stops after passing a certain distance. The initial domain wall shape is restored when the current is switched off.

The physical origin of spin torque stems from the exchange interaction between localized moments and conduction electrons. Thus, the spin accumulation induced by current is crucial for the spin torque exerted on the domain walls [10]. Hence, in this paper we calculate the current-induced (nonequilibrium) spin accumulation in a model ferromagnetic system with a relatively thick Néel domain wall. The approach is based on some unitary transformation, that has been used earlier to calculate the domain wall resistance [1]. The current-induced spin density is then calculated using linear response theory and the Green function formalism. In Sect. 2 we describe the model. Spin accumulation induced by current is calculated in Sect. 3, where also some numerical results are presented. Final conclusions are in Sect. 4 .

\section{Model}

We consider a ferromagnetic metal with a nonuniform magnetization corresponding to a single thick domain wall. The wall is described by the magnetization profile $\boldsymbol{M}(\boldsymbol{r})$. Assuming that $|\boldsymbol{M}(\boldsymbol{r})| \equiv M=$ const, one can write $\boldsymbol{M}(\boldsymbol{r})=M \boldsymbol{n}(\boldsymbol{r})$, where $\boldsymbol{n}(\boldsymbol{r})$ is a unit vector field describing spatial variation of the magnetization orientation.

The single-particle Hamiltonian describing conduction electrons which are locally exchange-coupled to the magnetization $\boldsymbol{M}(\boldsymbol{r})$ can be written in the form

$$
H_{0}=-\frac{\hbar^{2}}{2 m} \sum_{\alpha} \psi_{\alpha}^{\dagger} \frac{\partial^{2}}{\partial \boldsymbol{r}^{2}} \psi_{\alpha}-J \sum_{\alpha \beta} \psi_{\alpha}^{\dagger} \boldsymbol{\sigma}_{\alpha \beta} \cdot \boldsymbol{n}(\boldsymbol{r}) \psi_{\beta},
$$

where $J$ is the corresponding exchange parameter, $\psi_{\alpha(\beta)}$ and $\psi_{\alpha(\beta)}^{\dagger}$ are the spinor field operators for conduction electrons, and $\boldsymbol{\sigma}=\left(\sigma_{x}, \sigma_{y}, \sigma_{z}\right)$ represents the spin Pauli matrices. 
Our considerations are restricted to a specific model domain wall shape. First, we assume that the wall is translationally invariant in the $x-y$ plane, so $\boldsymbol{n}(\boldsymbol{r})$ depends only on the direction normal to the wall, $\boldsymbol{n}(\boldsymbol{r}) \rightarrow$ $\boldsymbol{n}(z)$. For a simple domain wall with $\boldsymbol{M}(z)$ changing in the plane normal to the wall (the Néel wall), one can parameterize the vector $\boldsymbol{n}(z)$ as

$$
\boldsymbol{n}(z)=(\sin \varphi(z), 0, \cos \varphi(z)),
$$

where the phase $\varphi(z)$ describes shape of the domain wall. For instance, if we assume the domain wall in the form of a kink, then

$$
\varphi(z)=-\frac{\pi}{2} \tanh (z / L),
$$

where $L$ is the domain wall width.

The first step of the analysis is to perform a unitary transformation to a local frame, $\psi \rightarrow T(z) \psi$ with $T^{\dagger}(z) T(z)=1$, which removes the inhomogeneity of $\boldsymbol{n}(z)$ [1]. In other words, $T(z)$ transforms the second term in Eq. (1) as

$$
T^{\dagger}(z) \boldsymbol{\sigma} \cdot \boldsymbol{n}(z) T(z)=\sigma_{z} .
$$

The transformed Hamiltonian in the adiabatic approximation, which is valid for thick domain walls, $L \gg \lambda$ (where $\lambda$ is the electron wavelength), can be written in the basis of semiclassical functions as [1]:

$$
\begin{aligned}
H_{0} & =\frac{\hbar^{2} k^{2}}{2 m}-J \sigma_{z} \\
& +\hbar^{2}\left[\frac{m \kappa^{2}(z)}{2}+\mathrm{i} \sigma_{y} \frac{\kappa^{\prime}(z)}{2}-\sigma_{y} \kappa(z) k_{z}\right],
\end{aligned}
$$

where $\kappa(z)=\varphi^{\prime}(z) /(2 m), \kappa^{\prime}(z) \equiv \partial \kappa(z) / \partial z$, whereas $\varphi^{\prime}(z) \equiv \partial \varphi(z) / \partial z$. When the domain wall is in the form of a kink, see Eq. (3), the parameter $\kappa(z)$ takes the form

$$
\kappa(z)=-\frac{\pi}{4 m L \cosh ^{2}(z / L)} .
$$

For a slowly varying smooth function $\varphi(z)$ (thick domain wall centered at $z=0$ ), the perturbation due to the domain wall is small and localized close to the center of the wall, $|z| \lesssim L$. The term proportional to $\kappa^{\prime}$ in Hamiltonian (5) is generally smaller than the other terms and may be ignored in further calculations.

As mentioned in the introduction, the domain wall leads to scattering of electrons, which in turn gives rise to excess spin density in the wall. The equilibrium (i.e., in the absence of external electric field) spin density of conduction electrons in the local reference frame can be calculated as

$$
\boldsymbol{S}=-\mathrm{i} \operatorname{Tr} \int \frac{\mathrm{d} \varepsilon}{2 \pi} \frac{\mathrm{d}^{3} k}{(2 \pi)^{3}} \boldsymbol{\sigma} G_{\varepsilon}(\boldsymbol{k}),
$$

where $G_{\varepsilon}(\boldsymbol{k})$ is the Green function corresponding to the Hamiltonian (5). In this paper, however, we are interested in the current-induced part of the spin density, which is responsible for the spin torque exerted on the domain wall. This part will be calculated in the following section.

\section{Spin accumulation and spin torque: linear response regime}

We assume now that the system is in an external field described by the time dependent vector potential $\boldsymbol{A}(t)=\boldsymbol{A}_{\omega} \exp (-\mathrm{i} \omega t)$. The electric field is then $\boldsymbol{E}(t)=-(1 / c)(\partial \boldsymbol{A}(t) / \partial t)=(\mathrm{i} \omega / c) \boldsymbol{A}_{\omega} \exp (-\mathrm{i} \omega t)$, or equivalently $\boldsymbol{E}_{\omega}=(\mathrm{i} \omega / c) \boldsymbol{A}_{\omega}$. Hamiltonian of the system in the vector field $\boldsymbol{A}(t)$ can be obtained by replacing $-\mathrm{i} \hbar(\partial / \partial \boldsymbol{r})$ in Eq. (5) by $-\mathrm{i} \hbar(\partial / \partial \boldsymbol{r})-(e / c) \boldsymbol{A}(t)$, where $e$ denotes electron charge $(e<0)$. We are considering only terms linear in $\boldsymbol{A}(t)$.

Now, we calculate the electron spin density induced by the external field $\boldsymbol{A}(t)$ in the linear response regime. Since we restrict ourselves to the adiabatic approximation (valid for small values of $\kappa(z)$ ), we will consider only the terms linear in the parameter $\kappa(z)$. Then, the current-induced spin density $\boldsymbol{S}$ can be calculated from the formula

$$
\boldsymbol{S}=\operatorname{Re}\left(-\mathrm{i} \operatorname{Tr} \int \frac{\mathrm{d} \varepsilon}{2 \pi} \frac{\mathrm{d}^{3} \boldsymbol{k}}{(2 \pi)^{3}} \boldsymbol{\sigma} G_{A \varepsilon}(\boldsymbol{k})\right),
$$

where the Green function $G_{A \varepsilon}(\boldsymbol{k})$ should be taken in the linear approximation with respect to the vector field $\boldsymbol{A}(t)$. From the perturbation expansion one finds [11]:

$$
G_{A \varepsilon}(\boldsymbol{k})=G_{\varepsilon+\hbar \omega}(\boldsymbol{k}) H_{A}(\boldsymbol{k}, \omega) G_{\varepsilon}(\boldsymbol{k}),
$$

where

$$
H_{A}(\boldsymbol{k}, \omega)=-\frac{\hbar e \boldsymbol{k} \cdot \boldsymbol{A}_{\omega}}{m c}+\frac{\hbar e A_{\omega}^{z}}{c} \kappa(z) \sigma_{y}
$$

is the perturbation linear in the vector field $\boldsymbol{A}_{\omega}$.

Since there are two different terms in $H_{A}$, see Eq. (10), we will calculate separately the corresponding contributions to the induced spin density. In the dc limit, the contribution to $\boldsymbol{S}$ from the first term in Eq. (10) is given by

$$
\begin{aligned}
& S_{x}^{(1)}=0, \\
& S_{y}^{(1)}=\frac{e \hbar E \kappa(z) \tau}{6 \pi^{2} J}\left(k_{\mathrm{F} \uparrow}^{3}-k_{\mathrm{F} \downarrow}^{3}\right),
\end{aligned}
$$

where $E$ is the electric field, while $k_{\mathrm{F} \uparrow}$ and $k_{\mathrm{F} \downarrow}$ are the Fermi wave vectors corresponding to the spin majority and spin minority electron subbands, respectively. We note that $k_{\mathrm{F} \uparrow}$ and $k_{\mathrm{F} \downarrow}$ are related to $J$ via the formula $2 J=\left(\hbar^{2} / 2 m\right)\left(k_{\mathrm{F} \uparrow}^{2}-k_{\mathrm{F} \downarrow}^{2}\right)$. Finally, $\tau$ is the electron relaxation time (assumed the same for both spin conduction channels).

Similarly, contribution to the spin density from the second term in the perturbation (10) acquires the form

$$
\begin{aligned}
& S_{x}^{(2)}=-\frac{e \hbar^{2} E \kappa(z)}{12 J^{2} \pi^{2}}\left(k_{\mathrm{F} \uparrow}^{3}-k_{\mathrm{F} \downarrow}^{3}\right), \\
& S_{y}^{(2)}=-\frac{e \hbar E m \kappa(z)}{8 \pi^{2} J^{2} \tau}\left(k_{\mathrm{F} \uparrow}+k_{\mathrm{F} \downarrow}\right) .
\end{aligned}
$$

Thus, the total spin density components are $S_{x}=S_{x}^{(1)}+$ $S_{x}^{(2)}$ and $S_{y}=S_{y}^{(1)}+S_{y}^{(2)}$. 
Owing to the exchange interaction, the current-induced spin density generates the spin torque. The in-plane and out-of-plane components of the spin torque are usually written as

$$
\begin{aligned}
& \boldsymbol{T}_{\|}=b_{j} \text { In } \times(\boldsymbol{n} \times \partial \boldsymbol{n} / \partial z), \\
& \boldsymbol{T}_{\perp}=c_{j} \text { In } \times \partial \boldsymbol{n} / \partial z,
\end{aligned}
$$

respectively, where $I$ is the current density (related to $E$ via the Drude formula). Taking into account Eqs. (11) to (16) one finds $b_{j}$ and $c_{j}$ in the form

$$
\begin{aligned}
b_{j} & =\frac{\hbar}{2 e}\left[\left(\frac{\tau_{\mathrm{ex}}}{\tau}\right)^{2} \frac{3}{4^{3} \pi^{2}}\left(1-\xi^{2}\right)(1+\xi)-1+\xi^{3}\right] \\
& /\left(1+\xi^{3}\right), \\
c_{j} & =-\frac{\hbar}{2 e} \frac{1}{4 \pi} \frac{\tau_{\mathrm{ex}}}{\tau} \frac{1-\xi^{3}}{1+\xi^{3}},
\end{aligned}
$$

where $\tau_{\mathrm{ex}}=2 \pi \hbar / J$ and $\xi$ is defined as $\xi=k_{\mathrm{F} \downarrow} / k_{\mathrm{F} \uparrow}$.

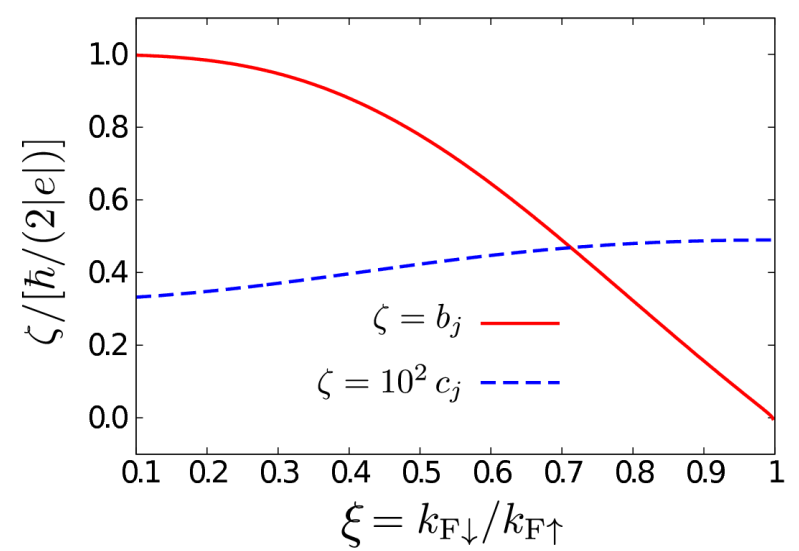

Fig. 1. Parameters $b_{j}$ and $c_{j}$ as a function of $\xi$ for $\mu=$ $10 \mathrm{eV}$ and $\tau=10^{-14} \mathrm{~s}$.

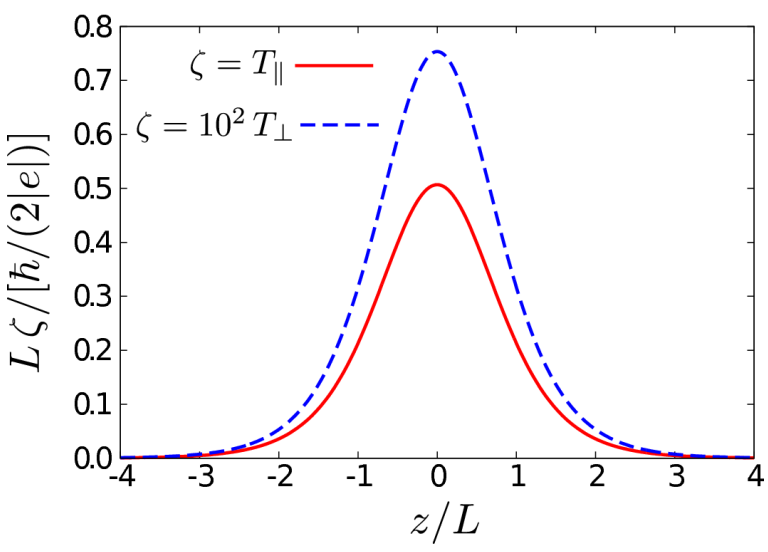

Fig. 2. Distribution of in-plane and out-of-plane components of spin torque along the normal to the domain wall for $\xi=0.8$. The other parameters as in Fig. 1 .

In Fig. 1 we show the parameters $b_{j}$ and $c_{j}$ as a function of $\xi$. The parameter $\mu$ is defined as the Fermi energy in the nonmagnetic state, $\mu=\left(\hbar^{2} k_{\uparrow(\downarrow)}^{2} / 2 m\right) \mp J$. In terms of $\xi$ this may be rewritten as $J=\left[\left(1-\xi^{2}\right) /\left(1+\xi^{2}\right)\right] \mu$. Let us note that the parameter $c_{j}$ is roughly two orders of magnitude smaller than $b_{j}$. The parameters $b_{j}$ and $c_{j}$ fully determine both in-plane and out-of-plane components of the spin torque. These components are maximal in the middle of the wall and decay as one departs from the wall center. This behavior is shown in Fig. 2 for $\xi=0.8$.

\section{Summary}

Using the Green function formalism and linear response theory we have calculated the current-induced spin accumulation in a Néel domain wall. The considerations are applicable to relatively thick domain walls, when the adiabatic approximation is justified. Such a situation takes place e.g. in metallic systems, especially in the bulk limit.

The current-induced spin density was used to find spin transfer torque exerted on the domain wall. The spin torque can be used to study current-induced domain wall dynamics, for instance via the Landau-Lifshitz-Gilbert equation.

\section{Acknowledgments}

This work was supported by the Polish Ministry of Science and Higher Education as research projects in years 2010-2011 (P.B.) and 2011-2014 (V.K.D.).

\section{References}

[1] V.K. Dugaev, J. Barnas, A. Lusakowski, L.A. Turski, Phys. Rev. B 65, 224419 (2002).

[2] J.C. Slonczewski, J. Magn. Magn. Mater. 159, L1 (1996).

[3] L. Berger, Phys. Rev. B 54, 9353 (1996).

[4] J.A. Katine, F.J. Albert, R.A. Buhrman, E.B. Myers, D.C. Ralph, Phys. Rev. Lett. 84, 3149 (2000).

[5] J. Grollier, P. Boulenc, V. Cros, A. Hamzič, A. Vaurès, A. Fert, G. Faini, Appl. Phys. Lett. 83, 509 (2003).

[6] A. Yamaguchi, T. Ono, S. Nasu, K. Miyake, K. Mibu, T. Shinjo, Phys. Rev. Lett. 92, 077205 (2004).

[7] M. Yamanouchi, D. Chiba, F. Matsukura, H. Ohno, Nature 428, 539 (2004).

[8] E. Saitoh, H. Miyajima, T. Yamaoka, G. Tatara, $N a-$ ture 432, 203 (2004).

[9] M. Kläui, C.A.F. Vaz, J.A.C. Bland, W. Wernsdorfer, G. Faini, E. Cambril, L.J. Heyderman, F. Nolting, U. Rüdiger, Phys. Rev. Lett. 94, 106601 (2005).

[10] S. Zhang, Z. Li, Phys. Rev. Lett. 93, 127204 (2004).

[11] A.A. Abrikosov, L.P. Gorkov, I. Ye. Dzyaloshinskii, Quantum Field Theoretical Methods in Statistical Physics, Pergamon Press, Oxford 1965. 